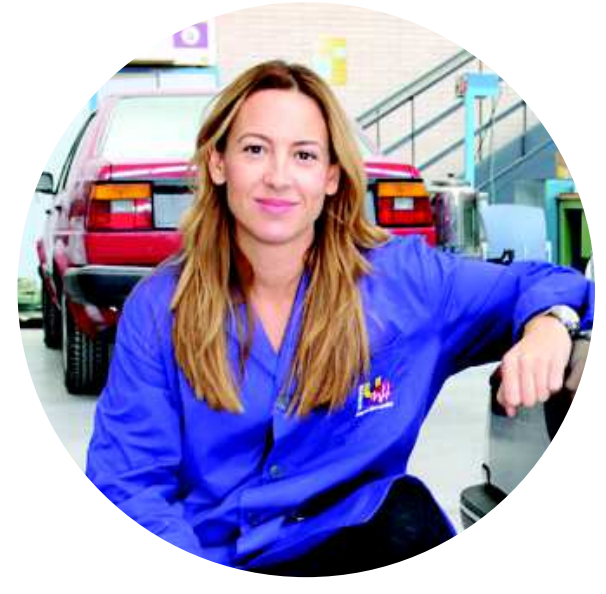

. Alicia de Lara

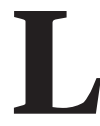

a ITV analiza los defectos que pueden darse en el coche, con el objetivo de garantizar la seguridad de la conducción. La profesora de la Universidad Miguel Hernández (UMH) de Elche Carolina Senabre investiga cómo el sistema de la ITV testa la capacidad de frenada sobre rodillos, "puesto que alrededor del 10\% de los defectos que se detectan en la ITV están relacionados con los frenos y con los neumáticos”, señala la profesora. La Asociación Española de Ingeniería Mecánica ha otorgado a Senabre el premio a la mejor Tesis Doctoral por su trabajo, titulado "Estudio y modelización del comportamiento de frenada de vehículos sobre banco de rodillos de ITV", dirigido por el profesor de la UMH Emilio Velasco Sánchez.

Se supone que los neumáticos del vehículo que acude a la ITV tienen la presión recomendada por el fabricante, pero en el manual de la ITV no existe un control de las ruedas antes de realizar la prueba de freno. De manera que esto queda en manos del conductor, cuando es algo que, según la investigación de Senabre, influye y puede hacer variar los datos obtenidos. Por ejemplo, la tesis apunta que para neumáticos del tipo Continental Contact e ITVs con unas características específicas, si los neumáticos tienen una presión de un bar, el vehículo no pasaría la prueba, mientras que si cuentan con una presión de tres bares sí pasaría sobradamente en las tres ITV utilizadas en el trabajo de campo. Este es uno de los parámetros que intervienen en la frenada y que puede variar los resultados, de todos los que cuentan en la medición y que han sido analizados en la tesis premiada.

\section{La frenada de vehículos sobre rodillos de ITV}

\author{
La profesora Carolina Senabre, \\ premio a la mejor tesis doctoral
}

Senabre demuestra con su trabajo que determinados parámetros, algunos incluso ajenos a la propia frenada del vehículo, influyen en su medición. Además de la presión en la rueda intervienen: tanto la distancia entre los rodiIlos (que puede variar hasta un I I\%), como la rugosidad de los rodillos del frenómetro (hasta un $27,7 \%$ ) -datos que dependen del banco de ITV al que se acceda- pero también el tipo de neumático, si es más rígido o más elástico (con un porcentaje de influencia en torno al 16\%). El peso sobre el eje también condiciona la frenada. Con incrementos de hasta 54 kilos sobre cada una de las ruedas, se observa que aumenta el valor de fuerza de frenada medida dependiendo, de nuevo, del tipo de neumático y de su presión, con diferencias de hasta un $12 \%$. Por último, el ángulo de convergencia y divergencia de las ruedas de un mismo eje también modifica la medida de frenada con diferencias, esta vez, de hasta un 10\%.

La prueba que realiza la ITV para testar los frenos consiste en situar el vehículo sobre un sistema de rodillos, donde los rodillos hacen girar la rueda por arrastre a $5 \mathrm{~km} / \mathrm{h}$, a continuación se pisa el pedal de freno hasta que se produce un deslizamiento total, momento en el que el vehículo es arrastrado fuera del sistema de rodillos. La normativa indica que para pasar la prueba el vehículo debe cumplir un valor mínimo de eficacia, fuerza de frenada dividido entre el peso, el mismo para todos los coches BI de España, que es del 50\%.

"Mi objetivo era encontrar un modelo matemático que reprodujera el movimiento de frenada de un vehículo sobre rodillos ya que, hasta el momento, nadie había modelizado esta curva”, señala la investigadora. Sobre plano sí existen diversos modelos matemáticos que indican cuál es la curva de deslizamiento, pero en la ITV se efectúa la prueba sobre rodillos por cuestiones de espacio, de practicidad y seguridad, en comparación con la pista.

Senabre se apoyó en la Fórmula de Pacejka para llevar a cabo su investigación, ya que la también conocida como 'fórmula mágica' le permitía utilizar datos experimentales y tener en cuenta todos los parámetros que entran en juego en la frenada longitudinal. "Se parte de que la frenada en pista y la de rodillos pueden aportar valores diferentes a la fórmula. La 


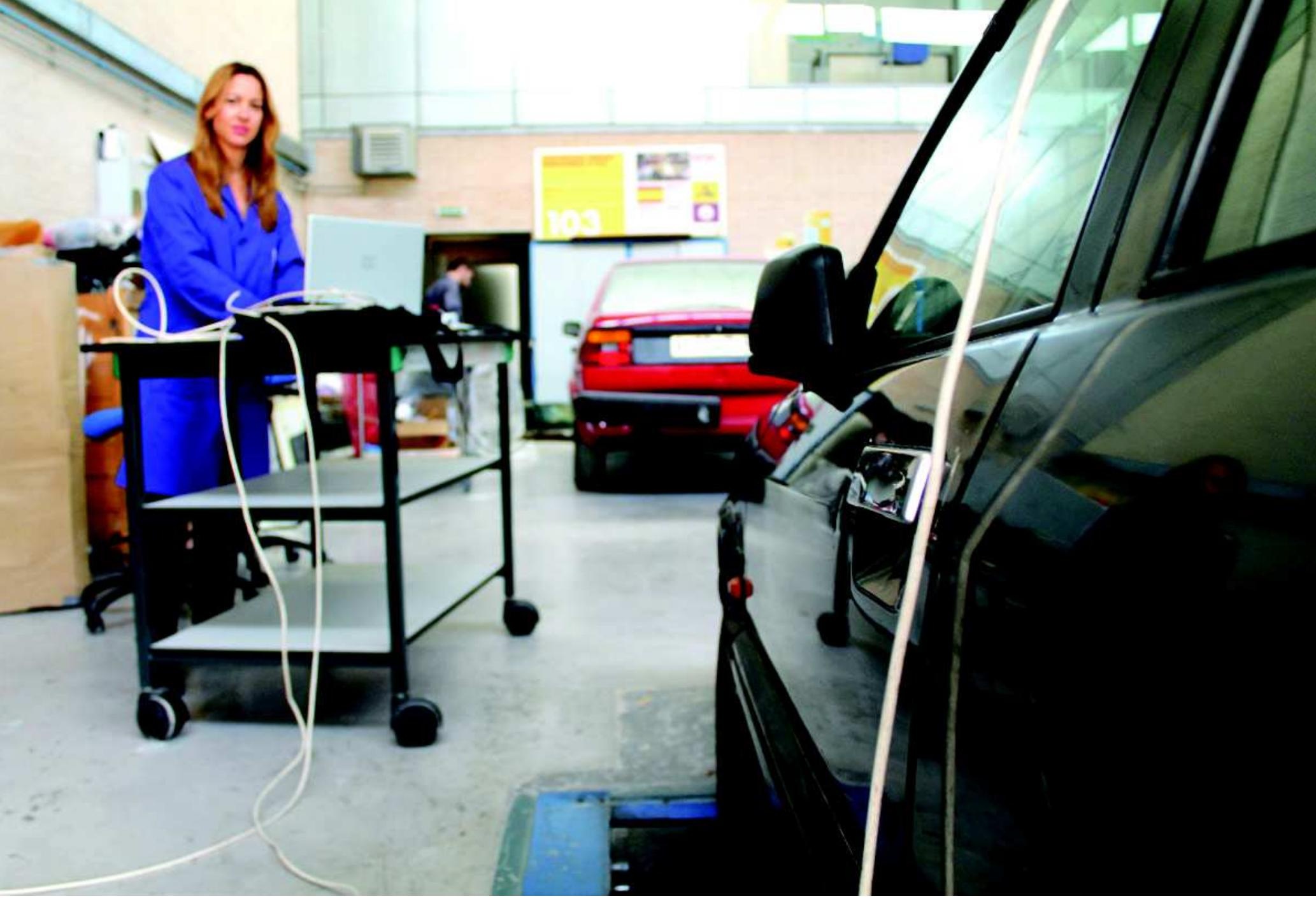

Carolina Senabre investiga en el Grupo de Ingeniería Mecánica Aplicada | Alicia de Lara

\section{Un modelo matemático inexistente hasta el momento}

pregunta es: ¿Cómo de diferentes? ¿Cómo varía en uno y otro caso la curva de frenada-deslizamiento?", se cuestionó la investigadora.

Para despejar el interrogante estudió todos los parámetros que entran en juego en la frenada sobre rodillos, que son distintos cuando se habla de frenada sobre rodillos o sobre pista. En concreto, aquellos que entran en juego solo en la frenada sobre rodillos son: la rugosidad de los rodillos y la separación existente entre los mismos, que puede variar de una planta a otra. El resto de parámetros que intervienen en ambos tipos de frenada son: la presión del neumático, su grado de desgaste y el tipo; la velocidad mínima que se debe alcanzar para deslizar el neumático; el ángulo de convergencia y divergencia existente entre las ruedas delanteras y la carga que soporta el eje.

El trabajo de campo se llevó a cabo en tres plantas de ITV diferentes -en las que variaba la distancia entre sus rodillos- y con el mismo vehículo para conocer la influencia de este parámetro. La profesora calibró los tres bancos con el mismo sistema de palanca y utilizó su propio sistema de adquisición de datos directamente del sensor de la ITV.

Además, ubicó un sensor de presión en el circuito de freno: "No solo se tenía en cuenta el grado de frenada que recibía el banco, sino también la que se producía en el sistema de frenos del vehículo", señala la investigadora. Así se obtuvo también datos referentes al rendimiento de frenada de las ITV. Se trataba por tanto de averiguar cuánto es capaz de medir el frenómetro de la ITV de lo que realmente se está frenando. De hecho, la investigadora señala que el sensor de frenos de la ITV recibe tan solo un $18 \%$ de la fuerza que se aplica en la rueda. De la misma manera, cuando se compara la frenada en pista con la de rodillo, en el mejor de los casos, se está recogiendo solo un $12 \%$ de la frenada real, según los datos de la investigadora. La causa de un porcentaje tan bajo es el deslizamiento que se produce entre la rueda y los rodillos. Para la medición del deslizamiento, Senabre utilizó dos sensores de "velocidad angular" o encoders, a diferencia del sistema que aplican los propios bancos de ITV, con un sensor óptico, menos preciso en comparación.

Se consideraron diferentes tipos de neumáticos, en los que también se modificó el desgaste. Senabre demostró que si utiliza un neumático más elástico, se adapta mejor a la forma del rodillo y el incremento de presión favorece a la disminución de deslizamiento, mientras que si es un neumático más rígido ocurre todo lo contrario. $Y$ respecto al parámetro de la rugosidad de los rodillos también se observó una influencia notable, hasta el punto de poder afirmar que cuando haya ITV muy desgastadas, cuya rugosidad sea muy baja, los vehículos tendrán más dificultades para pasar la prueba de frenos, debido al incremento de deslizamiento neumático-rodillo.

El objetivo de la investigación se cumplió al alcanzar las distintas curvas de frenada sobre rodillos basada en los datos obtenidos en la experimentación, lo que permitió a la profesora diseñar un modelo matemático inexistente hasta el momento. 